\title{
Deformation mechanisms of face-centered-cubic metal nanowires with twin boundaries
}

\author{
A. J. Cao ${ }^{\mathrm{a})}$ and Y. G. Wei $\mathrm{i}^{\mathrm{b})}$ \\ State Key Laboratory of Nonlinear Mechanics, Institute of Mechanics, Chinese Academy of Sciences, \\ Beijing 100080, People's Republic of China \\ Scott X. Mao ${ }^{\mathrm{c})}$ \\ Department of Mechanical Engineering and Materials Science, University of Pittsburgh, Pittsburgh, \\ Pennsylvania 15261
}

(Received 19 November 2006; accepted 8 March 2007; published online 11 April 2007)

\begin{abstract}
This letter addresses the issue of deformation mechanisms and mechanical tensile behavior of the twinned metal nanowires using atomistic simulations. Free surfaces are always the preferential dislocation nucleation sites in the initial inelastic deformation stage, while with further plastic deformation, twin boundary interfaces will act as sources of dislocations with the assistance of the newly formed defects. The smaller the twin boundary spacing, the higher the yielding stresses of the twinned nanowires. Twin boundaries, which serve both as obstacles to dislocation motion and dislocation sources, can lead to hardening effects and contribute to the tensile ductility. This work illustrates that the mechanical properties of metal nanowires could be controlled by tailoring internal growth twin structures. (C) 2007 American Institute of Physics. [DOI: 10.1063/1.2721367]
\end{abstract}

Due to the unique mechanical, electrical, and optical properties, materials with nanometer-sized structure have attracted a great deal of interest in the past few decades ${ }^{1-3}$ Many researchers have demonstrated, through both experiments and analysis, that the structure and properties of nanowires can be quite different from those of bulk materials due to the effects of larger free surfaces. ${ }^{4-14}$ Simulations reported in the previous literatures are generally defect-free and the sizes of the modeled wires are usually smaller than $6 \mathrm{~nm}$. $^{6-14}$ Meanwhile experimentally, twins, a class of planar defects, are observed most often in single-crystal metal (copper, silver, and gold) nanowires with a [111] growth orientation. ${ }^{15,16}$ Because twin boundaries (TBs) in nanowires will strongly affect the physical properties of nanowires, it is of great significance to investigate the details of twin structures and their roles in metal nanowires. Until now, there are still some key issues about twin related deformation which are not quite clear and need fundamental understanding. For example, what is the role of TBs in mechanical deformation? Does it act as grain boundaries or surfaces? In our recent work, the mechanical behavior of fivefold twinned nanowires is studied. ${ }^{17}$ We found that the strengthening mechanism in the fivefold twinned nanowires is due to the TBs as obstacles to the dislocation motion. In this letter, we address the effect of TBs on inelastic deformation of the twinned nanowires using atomistic simulations.

In this work we focus on twinned $\mathrm{Cu}$ nanowires with 〈111) growth orientation and nearly square cross section as indicated from the experimental observations. ${ }^{15,16}$ The initial configuration of the twinned nanowires is constructed by repeating $\Sigma 3$ coherent twins in the $\langle 111\rangle$ axis orientation. ${ }^{18}$ Four samples are prepared for the simulations. The first one consists of two twins, the second consists of four twins, and the third contains five twins. The twin boundary spacings (TBSs) of the first, second, and third wires are $14 \mathrm{~nm}$

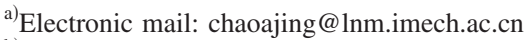

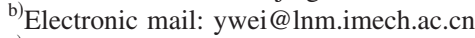

${ }^{c)}$ Electronic mail: smao@engr.pitt.edu
}

(sample I), $7 \mathrm{~nm}$ (sample II), and $5 \mathrm{~nm}$ (sample III), respectively. The fourth twin-free wire (sample IV) oriented in the [111] direction with nearly square cross section and ( $\overline{1} \overline{1} 2)$ and $(1 \overline{10})$ lateral surfaces is created corresponding to the bulk face-centered-cubic (fcc) crystal lattice. The undeformed configurations of these four samples are shown in Fig. 1. The sizes of the wires are the same for all these nanowires, which are $8 \times 8 \times 32 \mathrm{~nm}^{3}$. Up to 175000 atoms are contained in the simulation model. An embedded-atom method potential for $\mathrm{Cu}$ by Mishin et al. ${ }^{19}$ is chosen in the present simulations because it is calibrated according to the $a b$ initio values of stacking fault and twin formation energies. $^{19}$

To discern defects in the nanowires, colors are assigned to the atoms according to a local crystallinity classification visualized by common neighbor analysis, ${ }^{20}$ which permits the distinction between atoms in a local hexagonal-close- (a)

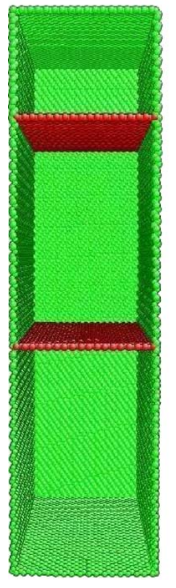

(b)

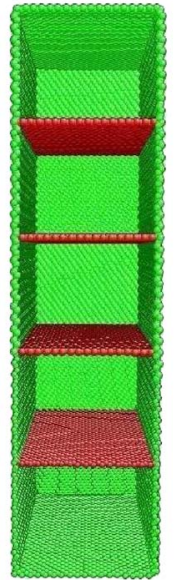

(c)

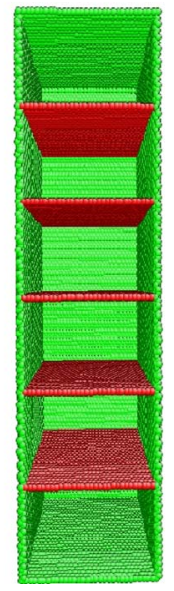

(d)

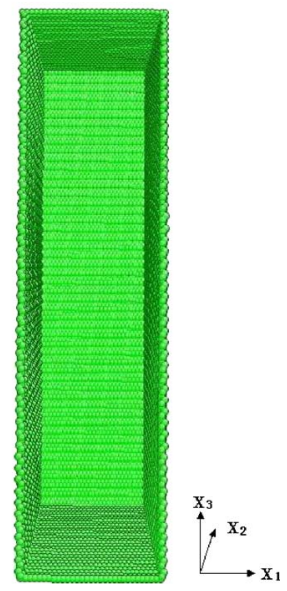

FIG. 1. (Color online) Undeformed configurations of (a) nanowire with two twins, (b) nanowire with four twins, (c) nanowire with five twins, and (d) twin-free nanowire. TB is shown with one hcp plane in the nanowire interior. Perfect fcc atoms and the front surface are not shown for viewing inner TBs. 
packed (hcp) environment and those in a fcc environment. Gray stands for fcc atoms, red for hcp atoms, green for other 12 coordinated atoms, and blue for the non-12-coordinated atoms. A single line of hcp atoms represents a twin boundary, two adjacent hcp lines stand for an intrinsic stacking fault, and two hcp lines with a fcc line stand for an extrinsic stacking fault between them. Other 12 coordinated atoms and non-12-coordinated atoms appear in the free surface region and in the core of dislocations. This is the same method used in Refs. 17 and 21.

After the initial construction, conjugate gradient method is used to minimize the whole wire energy to obtain equilibrium configurations. And then the wires are thermally equilibrated to $300 \mathrm{~K}$ for $20 \mathrm{ps}$ using a Nosé-Hoover thermostat $^{22,23}$ while keeping the lengths of the wires constant. Free boundary conditions are utilized in all three dimensions in the whole work.

Starting from the equilibrium configuration of the nanowires, uniaxial tensile loading is applied until failure under the simulated quasistatic conditions. ${ }^{7,12,17}$ To accelerate simulation efficiency, in the first seven loading steps, all the atoms are first displaced in a prescribed uniform strain increment of $0.5 \%$ in the length direction, and $0.2 \%$ strain increment is applied in the remaining steps. The wires are then relaxed with their ends fixed at a constant temperature of $300 \mathrm{~K}$ for $25 \mathrm{ps}$ at their terminals to obtain a macroscopic equilibrium configuration. The equilibrated process usually takes less than $15 \mathrm{ps}$, and the average stress over the last $5 \mathrm{ps}$ of the relaxation period is taken as the stress of the nanowire. The stresses reported in this work are calculated using the Virial theorem, which is equivalent to the Cauchy stress in the average sense. ${ }^{24}$

Tensile stress-strain curves for the four simulated nanowires are shown in Figs. 2(a) and 2(b) for large-scale and small-scale (local) horizontal coordinates, respectively. Upon tensile loading, the four wires show linear increase in stress with increasing strain in the elastic deformation stage [Fig. 2(b)]. Young's modulus calculated from the stress-strain curve is about $190 \mathrm{GPa}$ for the $\langle 111\rangle$ type crystalline directions and the results from the four samples display little difference. After the elastic deformation, the stress-strain curves show a precipitous drop, which implies that yielding has occurred in the nanowires. The yielding stresses of the twotwin nanowire, four-twin nanowire, and five-twin nanowire are 6.9, 7.6, and $8.0 \mathrm{GPa}$, respectively, while the yielding stress of the twin-free wire is $6.3 \mathrm{GPa}$. One can find that there is an increase in the strength of the twinned sample for the twin-free sample, $10 \%$ for sample I, $21 \%$ for sample II, and $27 \%$ for sample III. The yielding stress of wires is inversely proportional to the TBS. This strongly indicates that TBS plays a significant role in the strengthening of the nanowires. From Fig. 2(a), one can find that the ductility of the twinned nanowires increases with increasing the TBS, while the ductility is lowest for the case of the twin-free nanowire. One kind of interpretations for these phenomena is attributed to the ample room for dislocation storage in the large TBS wire, while a little dislocation activity is available in the small TBS wire beacause the motion of dislocations along the well-defined slip systems is restricted by TBs. In contrast, in the twin-free wire, the glide of dislocations on the slip planes would cause steps and jogs at the side surfaces, which are preferred sites for necking failure. From the above results, one can reach an important conclusion that the TBs Downloaded 13 Jun 2010 to 159.226.231.78. Redistribution subject
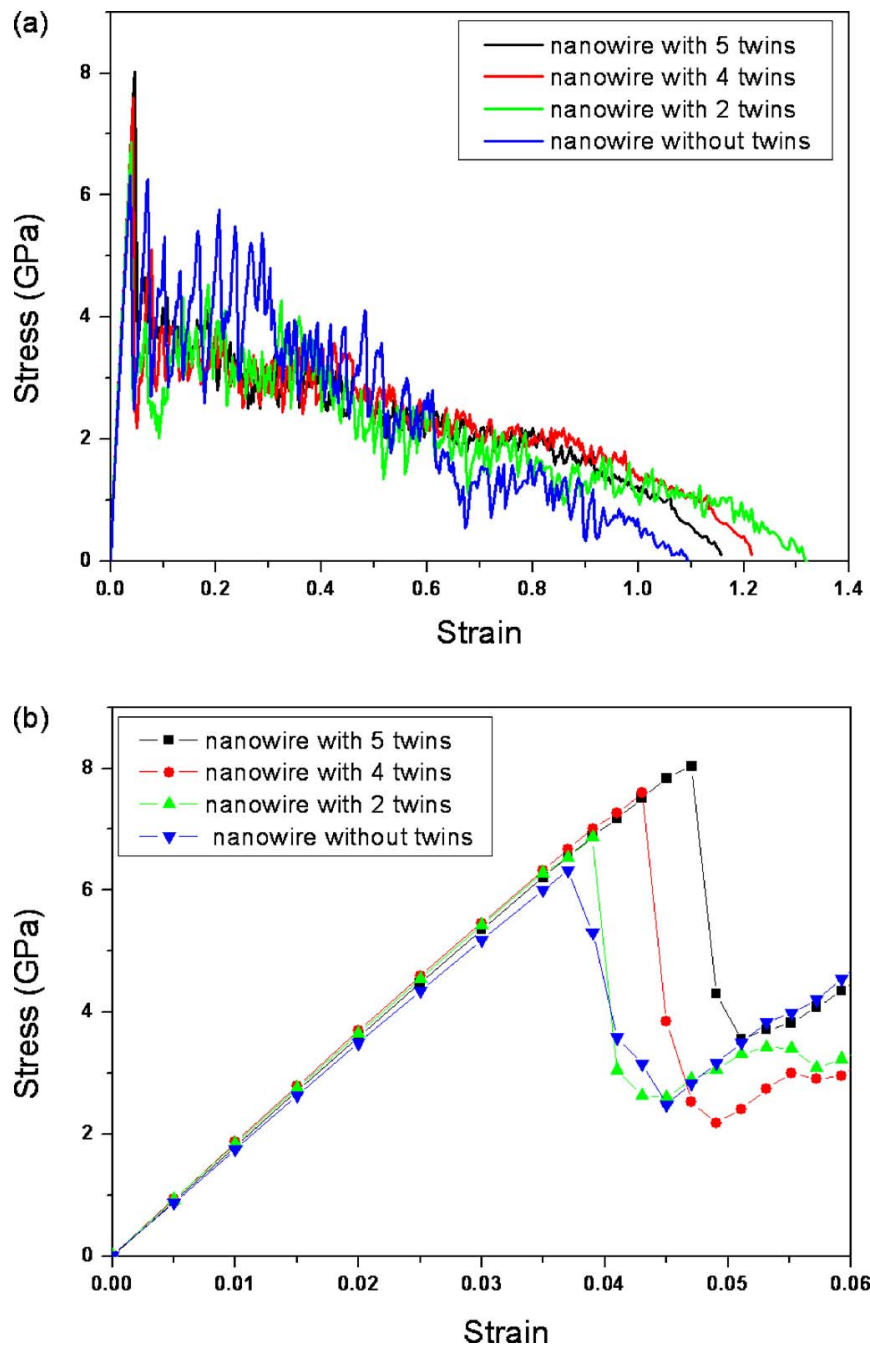

FIG. 2. (Color online) (a) Tensile stress-strain curves for the four simulated nanowires (large-scale horizontal coordinate). (b) Tensile yielding stressstrain curve for the four simulated nanowires (small-scale horizontal coordinate).

can promote both the strength and the tensile ductility, so the controlled mechanism related to the twinned structure would have the potential to develop a high-strength and highductility metal nanowire.

Upon tensile loading of the $\langle 111\rangle$ growth twinned nanowires, the first leading partial dislocation is nucleated from edge of the free surface, and then the movement of dislocation is blocked by TBs; after several picoseconds late the trailing partial dislocation is emitted from the same surface edge. A dynamic observation of the typical dislocation activities during the initial yielding deformation of sample II is shown in Fig. 3. The trapped dislocations cannot egress out of the wire due to the presence of TBs, while in the twin-free wire, dislocations glide across the whole wire and incorporate in the opposite site of the side surfaces and no dislocations are left in the wire. With further tensile deformation, another partial dislocation is emitted from the other site of the free surfaces and is blocked by TBs, as shown in Figs. 3(c)-3(e).

Dislocation loops emitted from TB interfaces, usually in response to other dislocations imprinting on the TB interfaces, are interestingly observed in our simulations. The dislocations trapped by TBs and roughening of the TBs could form a new step in the vicinity of the TBs, which assists the to AIP license or copyright; see http://apl.aip.org/apl/copyright.jsp 
(a)

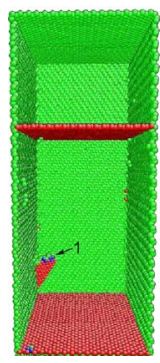

(b)

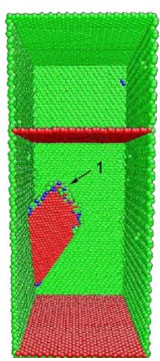

(c)

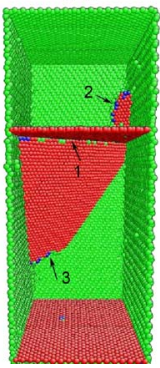

(d)

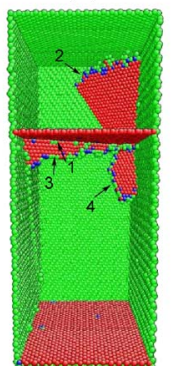

(e)

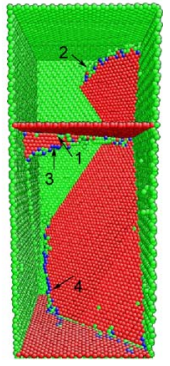

FIG. 3. (Color online) Continuous snapshots of initial yielding deformation of two-twin nanowire. (a) Reference time: $0 \mathrm{ps}$, first leading partial 1 is nucleated from the surface edge with stacking faults left behind. (b) $2.5 \mathrm{ps}$, partial 1 glides on the $\{111\}$ slip plane. (c) $14.5 \mathrm{ps}$, trailing partial 3 is nucleated and propagates toward TB and partial 2 is nucleated from the free surface. (d) $24.5 \mathrm{ps}$, trailing partial 3 pils up at TB and partial 4 is nucleated from the other site of the free surface. (e) $29 \mathrm{ps}$, partial 4 propagates and then trapped by TB. Perfect fcc atoms and the front surface are not shown for viewing inner defects. Only the upper parts of the nanowire are shown TB is represented by one red hcp plane. Black arrows indicate Shockley partial dislocations.

dislocation nucleation from TBs. In other words, when the plastic deformation is quite large, TBs gradually lose their coherency and then can be served as dislocation sources in the twinned nanowires. An example of a half-loop of partial dislocation emitted from TB and then trapped by other TBs and side free surfaces is shown in Fig. 4. The abovementioned mechanisms of dislocation emitted from TBs are similar to the experimental observations, in which emission of dislocation half-loops from the $\mathrm{Al} / \alpha-\mathrm{Al}_{2} \mathrm{O}_{3}$ epitaxial interface of thermally strained nanowires is detected by the in

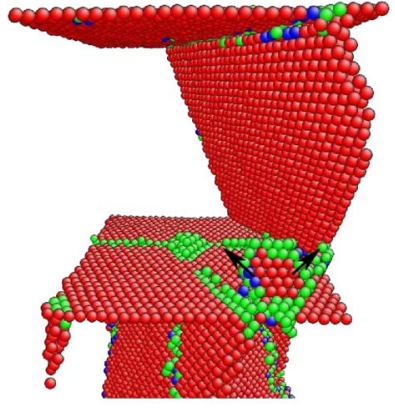

(a)

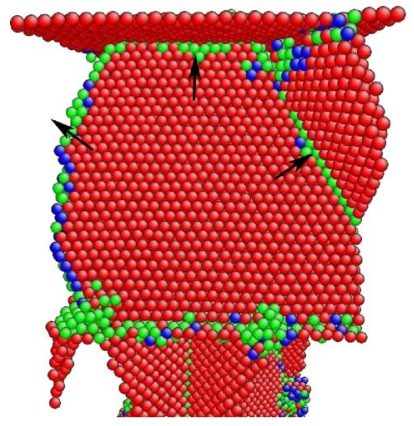

(c)

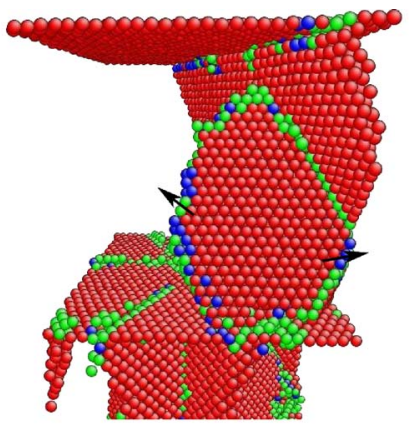

(b)

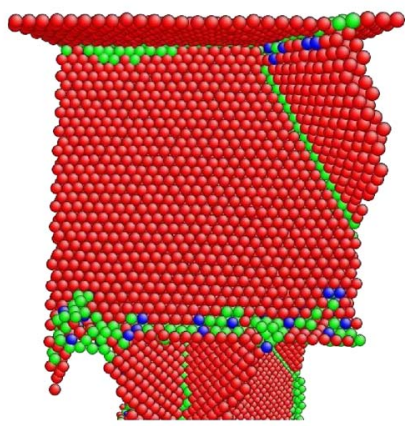

(d)
FIG. 4. (Color online) Typical new half-loop of partial dislocation emission from TB. (a) Partial dislocation half-loop nucleus (embryo) is nucleated from the TB with the assistance of some trapped dislocations and steps, (b) the dislocation loop grows, [(c) and (d)] dislocation intersects with upper TB, stacking fault, and side surfaces. Side surfaces are not shown for viewing interior defects. Black arrows indicate dislocation growth direction. situ transmission electron microscope ${ }^{25}$ Recently, the role of TBs in tensile deformation of different nanocrytalline materials is investigated using molecular dynamics (MD) simulations. ${ }^{26-28}$ The vicinal TBs providing dislocations sites are reported in nanocrystalline $\mathrm{Al}$, in which even the presence of one atomic plane step structure can trigger dislocation activity sources. ${ }^{28}$ This is similar to the results observed in the present work.

In summary, we have performed atomistic simulations on the tensile deformation of the $\langle 111\rangle \mathrm{Cu}$ nanowires with and without the presence of TBs. Based on the simulated results, TBs play a vital role in the deformation mechanisms and therefore fracture properties. The simulations have shown the following (1) Free surfaces are the preferential dislocation nucleation sites in the early inelastic deformation stage due to the effects of free surfaces. (2) The smaller the TBS, the higher the twinned nanowire yield stress. The redistribution of interior stress owing to the presence of TBs is responsible for the strengthening of the twinned nanowires. (3) TBs act as barrier for dislocation movements and consequently lead to hardening effects. (4) TBs can be available as dislocation sources with further straining, especially with the assistance of the newly formed defects on TBs, which can contribute to tensile ductility. This study demonstrates that the mechanical properties of metal nanowires can be controlled by tailoring interior growth twin structures.

The authors are supported by the National Science Foundation of China through Grant Nos. 10432050, 10428207, and 10672163; KJCX2-YW-M04 of the Chinese academy of Sciences.

${ }^{1}$ E. W. Wong, P. E. Sheehan, and C. M. Lieber, Science 277, 1971 (1997).

${ }^{2}$ A. Hasmy and E. Medina, Phys. Rev. Lett. 88, 096103 (2002).

${ }^{3}$ E. Z. da Silva, A. J. R. da Silva, and A. Fazzio, Phys. Rev. Lett. 87, 256102 (2001)

${ }^{4}$ Y. Kondo and K. Takayanagi, Phys. Rev. Lett. 79, 3455 (1997).

${ }^{5}$ Y. Kondo and K. Takayanagi, Science 289, 606 (2000).

${ }^{6}$ W. Liang, M. Zhou, and F. Ke, Nano Lett. 5, 2039 (2005).

${ }^{7}$ W. Liang and M. Zhou, Phys. Rev. B 73, 115409 (2006).

${ }^{8}$ H. S. Park, K. Gall, and J. A. Zimmerman, Phys. Rev. Lett. 95, 255504 (2005).

${ }^{9}$ J. Diao, K. Gall, and M. L. Dunn, Nat. Mater. 2, 656 (2003).

${ }^{10}$ J. Diao, K. Gall, and M. L. Dunn, Nano Lett. 4, 1863 (2004).

${ }^{11}$ J. Diao, K. Gall, M. L. Dunn, and J. A. Zimmerman, Acta Mater. 54, 643 (2006).

${ }^{12}$ K. Gall, J. Diao, and M. L. Dunn, Nano Lett. 4, 2431 (2004).

${ }^{13}$ W. Liang and M. Zhou, J. Eng. Mater. Technol. 127, 423 (2005).

${ }^{14}$ H. S. Park and J. A. Zimmerman, Phys. Rev. B 72, 054106 (2005).

${ }^{15}$ M. L. Tian, J. G. Wang, J. Kurtz, T. E. Mallouk, and M. H. W. Chan, Nano Lett. 3, 919 (2003)

${ }^{16}$ J. G. Wang, M. L. Tian, T. E. Mallouk, and M. H. W. Chan, J. Phys. Chem. B 108, 841 (2004).

${ }^{17}$ A. J. Cao and Y. G. Wei, Phys. Rev. B 74, 214108 (2006).

${ }^{18}$ J. M. Howe, Interfaces in Materials (Wiley, New York, 1997), Chap. 13, pp. 333-337.

${ }^{19}$ Y. Mishin, M. J. Mehl, D. A. Papaconstantopoulos, A. F. Voter, and J. D. Kress, Phys. Rev. B 63, 224106 (2001).

${ }^{20}$ J. D. Honeycutt and H. C. Andersen, J. Phys. Chem. 91, 4950 (1987).

${ }^{21}$ A. J. Cao and Y. G. Wei, Appl. Phys. Lett. 89, 041919 (2006).

${ }^{22}$ S. Nosé, J. Chem. Phys. 81, 511 (1984).

${ }^{23}$ W. G. Hoover, Phys. Rev. A 31, 1695 (1985).

${ }^{24}$ K. S. Cheung and S. Yip, J. Appl. Phys. 70, 5688 (1991).

${ }^{25}$ B. J. Inkson, G. Dehm, and T. Wagner, Acta Mater. 50, 5033 (2002).

${ }^{26}$ A. G. Frøseth, H. Van Swygenhoven, and P. M. Derlet, Acta Mater. 52, 2259 (2004).

${ }^{27}$ A. G. Frøseth, P. M. Derlet, and H. Van Swygenhoven, Appl. Phys. Lett. 85, 5863 (2004)

${ }^{28}$ A. G. Frøseth, P. M. Derlet, and H. Van Swygenhoven, Scr. Mater. 54, 477 (2006). 